\title{
Análise espacial e temporal da cobertura da triagem auditiva neonatal no Brasil (2008-2015)
}

\author{
Spatial and temporal analysis of the coverage \\ for neonatal hearing screening in Brazil (2008-2015)
}

Monique Ramos Paschoal ${ }^{1}$
Hannalice Gottschalck Cavalcanti $^{2}$
Maria Ângela Fernandes Ferreira

${ }^{1}$ Departamento de Odontologia, Centro de Ciências da Saúde, Universidade Federal do Rio Grande do Norte. Av. Senador Salgado Filho 1787, Lagoa Nova. 59056-000 Natal RN Brasil.moniquerpaschoal@ gmail.com ${ }^{2}$ Departamento de Fonoaudiologia, Universidade Federal da Paraíba. João Pessoa PB Brasil.

\begin{abstract}
This article seeks to establish the coverage of neonatal hearing screening in Brazil between January 2008 and June 2015. It is an ecological study that uses the country, through the Urban Articulation Regions, as a base. To calculate the screening coverage percentage, the Live Births Information System, the Outpatient Information System and the Beneficiaries of the National Supplementary Health Agency Information System were used. An exploratory analysis of maps and spatial statistical analysis was conducted using TerraView 4.2.2 software. The coverage of neonatal hearing screening saw an increase of $9.3 \%$ to $37.2 \%$ during the study period. In 20082009 it was observed that the percentage of coverage ranged from $0 \%$ to $79.92 \%$, but most areas received coverage from $0 \%$ to $20 \%$, though in 2014-2015 coverage ranged from $0 \%$ to $171.77 \%$, and there was a visible increase in the percentage of coverage in the country, mainly in the Southern Region. The screening coverage has increased over time, but is still low with an uneven distribution in the territory, which may be explained by local laws and policies and by the existence of different types of auditory health service in the country. Key words Hearing, Hearing loss, Neonatal screening, Unified Health System, Information systems
\end{abstract}

Resumo O objetivo deste artigo é conhecer a cobertura da triagem auditiva neonatal no Brasil de janeiro de 2008 a junho de 2015. Trata-se de um estudo ecológico que utiliza como base o território nacional, através das Regiões de Articulação Urbana. Para o cálculo da porcentagem da cobertura da triagem foram utilizados o Sistema de Informação de Nascidos Vivos, o Sistema de Informações Ambulatoriais e o Sistema de Informações de Beneficiários da Agência Nacional de Saúde Suplementar. Foi realizada a análise exploratória dos mapas e a análise espacial estatística por meio do programa TerraView 4.2.2. A cobertura da triagem auditiva neonatal apresentou uma evolução de 9,3 para 37,2\% no período estudado. Em 2008-2009 observa-se que a porcentagem da cobertura variou de 0,00 a 79,92\% e a maioria das regiões obteve cobertura entre 0,0 e 20\%, já em 2014-2015 a cobertura variou entre 0,0 a $171,77 \%$ e observou-se um visivel aumento da porcentagem da cobertura no país, principalmente na Região do Sul. A cobertura da triagem tem crescido ao longo do tempo, mas ainda é baixa e apresenta uma distribuição desigual no território, o que pode ser explicado pelas leis e politicas locais e pela disposição das diferentes modalidades de serviço de saúde auditiva no país.

Palavras-chave Audição, Perda auditiva, Triagem neonatal, Sistema Único de Saúde, Sistemas de informação 


\section{Introdução}

A triagem auditiva neonatal (TAN) é o meio mais efetivo e recomendado para propiciar a detecção e a intervenção precoce da perda auditiva em recém-nascidos, para que a perda não interfira de forma negativa no desenvolvimento da criança e posteriormente na sua qualidade de vida ${ }^{1}$.

Quando as alterações auditivas são diagnosticadas até os três meses de idade e a intervenção terapêutica é iniciada até os seis meses, o desenvolvimento da compreensão e da expressão da linguagem, bem como o relacionamento social e o desempenho acadêmico da criança com perda auditiva, pode ser compatível com o de crianças ouvintes da mesma faixa etária ${ }^{2}$.

Seguindo essa linha, a triagem deve ser a primeira etapa de um programa, realizada preferencialmente antes de um mês de vida, seguida de atendimento multidisciplinar para diagnóstico até os três meses e caso confirmada a perda auditiva, devem ser iniciados os processos de intervenção, com uso de amplificação sonora e reabilitação antes dos seis meses ${ }^{1}$.

A prevalência da perda auditiva congênita foi estimada em 1 a 3 para cada 1000 nascidos vivos e 2 a 4 para cada 100 nascidos vivos provenientes de Unidade de Terapia Intensiva Neonatal (UTIN) $)^{3}$. No Brasil, não há estudos de base populacional que determinam a prevalência da perda congênita, por isso os estudos internacionais são usados como estimativas na população brasileira.

Diante das estimativas e das consequências da privação sensorial na qualidade de vida do ser humano, a perda auditiva na infância configurase como um problema de saúde pública e justifica-se a realização da triagem de forma universal, para abranger todos os recém-nascidos.

Na década de 1990 no Brasil, surgiu uma preocupação maior com a triagem auditiva neonatal, foram criados serviços de atendimento e projetos de lei para tornar obrigatória a realização do procedimento. De 1998 a 2004, 13 municípios tiveram projetos de lei que foram aprovados sob diferentes abrangências, até que a vigência da Lei Federal 12.303/10 tornou obrigatória a realização gratuita do exame Emissões Otoacústicas Evocadas, em todos os hospitais e maternidades, nas crianças nascidas em suas dependências ${ }^{4,5}$.

Paralelo a essa evolução das políticas brasileiras voltadas à realização da triagem auditiva neonatal, estudos internacionais foram publicados e serviram para a elaboração de documentos com recomendações acerca do tema, como por exem- plo o uso das emissões otoacústicas (EOA) e do Potencial Evocado Auditivo de Tronco Encefálico (PEATE).

No Brasil, atualmente existem duas publicações importantes, a primeira foi emitida pelo Comitê Multiprofissional de Saúde Auditiva ${ }^{6}$, com intuito de nortear as ações dos profissionais envolvidos nos programas de triagem, no âmbito da prevenção, do diagnóstico e da reabilitação da perda auditiva e a segunda foi publicada pelo Ministério da Saúde, é a cartilha das Diretrizes de Atenção a Triagem Auditiva Neonatal com objetivo de melhorar a cobertura da triagem quanti e qualitativamente ${ }^{7}$.

$\mathrm{Na}$ literatura existe um consenso quanto à importância da universalidade da triagem, e que seus índices sejam superiores a 95\% dos nascidos vivos, porém a realidade do Brasil está muito aquém desse valor. $\mathrm{O}$ último estudo publicado estimou que em 2011 a cobertura alcançou $21,8 \%$ dos nascidos vivos e que existem fortes desigualdades inter e intrarregionais no país ${ }^{8}$.

Dentro desse contexto, reforça-se a necessidade de discussões científicas sobre a universalização do acesso e a busca pela equidade na cobertura da triagem auditiva no Brasil. Este estudo tem como objetivos realizar a análise espacial e temporal da cobertura de triagem auditiva neonatal dos usuários do Sistema Único de Saúde do Brasil no período de janeiro de 2008 a junho de 2015, avaliar se a legislação está determinando essa cobertura e verificar se a presença das diferentes modalidades de serviço de saúde auditiva coincide com uma maior cobertura.

\section{Métodos}

Trata-se de um estudo ecológico que utiliza como base o território Nacional. O estudo foi realizado com dados secundários agregados dos municípios brasileiros. O Instituto Brasileiro de Geografia e Estatística (IBGE) publicou o caderno Regiões de influência das cidades, com o objetivo de conhecer os relacionamentos entre as cidades brasileiras com base na análise dos fluxos de bens e serviços. Uma das divisões são as Regiões Intermediárias de Articulação Urbana, são regiões que possuem a capacidade de polarizar um número grande de municípios no atendimento a bens e serviços de alta complexidade e concentram e articulam atividades de gestão pública e privada, na escala regional. Existem 161 regiões no país9. O motivo da escolha dessa unidade foi porque permite analisar a cobertura do Brasil de forma mais 
minuciosa. A população de referência foram os nascidos vivos usuários do SUS no período de janeiro de 2008 a junho de 2015. Foram utilizados como fonte de dados o Sistema de Informação de Nascidos Vivos (SINASC), o Sistema de Informações Ambulatoriais (SIA/SUS) e o Sistema de Informações de Beneficiários (SIB) da Agência Nacional de Saúde Suplementar (ANS). Como o SINASC não possui os dados dos nascidos vivos de janeiro a junho de 2015, foi realizada uma estimativa utilizando os referentes a janeiro e junho de 2014, pois acredita-se que seja o mais próximo do real.

O levantamento dos dados foi realizado a partir da plataforma DATASUS, no link "Informações à saúde", restringindo a procura a "procedimento ambulatorial por local de atendimento" e tendo como abrangência geográfica o Brasil discriminado por unidade de análise "município" e como período "janeiro de 2008 a junho de 2015". O procedimento selecionado foi "Emissões Otoacústicas para triagem auditiva”, cujo código é 0211070149 . Em seguida, na plataforma DATASUS, no link “estatísticas vitais” e na opção "nascidos vivos" foi selecionada a mesma unidade de análise e o mesmo período, bem como a opção "nascim p/ resid.mãe".

Além disso, no SIB/ANS, foi identificada a cobertura total da assistência suplementar de saúde, através da quantidade de crianças até 1 ano cobertas por plano de saúde de janeiro de 2008 a junho de 2015 por município, para eliminá-las no cálculo da parcela da população usuária do SUS, devido à grande variação no território nacional. Dado que mais se aproxima da realidade nacional e do objetivo do estudo.

Os dados foram tabulados a partir do software Tabwin, disponibilizado pelo Departamento de Informática do Ministério da Saúde. A cobertura da TAN para nascidos vivos usuários SUS foi calculada através da seguinte fórmula: Porcentagem da cobertura da triagem auditiva neonatal $=\mathrm{nTA} / \mathrm{nNV}-\mathrm{nPS}$, onde nTA corresponde ao número de EOA para Triagem Auditiva aprovadas pelo SUS, nNV corresponde ao número de Nascidos Vivos e nPS corresponde à população coberta por Plano de Saúde. Essa porcentagem foi calculada por Regiões Intermediárias de Articulação Urbana.

Os dados foram divididos em quatro períodos de acordo com marcos importantes na literatura da triagem auditiva neonatal no Brasil. Foram os seguintes: Primeiro mapa (antes da Lei Federal no 12.303): jan/08 a dez/09; Segundo mapa (Lei Federal $n^{\circ} 12.303$ sancionada): jan/10 a dez/11;
Terceiro mapa (Publicação das Diretrizes): jan/12 a dez/13; Quarto mapa (atual): jan/14 a jun/15.

Foi calculada a porcentagem da cobertura da triagem auditiva neonatal nos quatro períodos por Regiões Intermediárias de Articulação Urbana, conforme descrito no tópico acima.

As variáveis independentes foram os serviços de saúde auditiva segundo as modalidades: Centros de Implante Coclear, Centros de Média Complexidade, Centros de Alta Complexidade e Centros de Reabilitação de Modalidade Auditiva. São serviços habilitados no Cadastro Nacional de Estabelecimentos de Saúde (CNES), no período de acesso ao site, janeiro de 2016.

Os dados foram compilados e organizados em um banco de dados com uso do programa Excel ${ }^{\circledR}$, em seguida realizado o Linkage das planilhas e agregação dos municípios em RAU's no software estatístico Statistical Package for the Social Sciences - SPSS ${ }^{\circledR}$ 18.0. Através do programa TerraView 4.2.2, foi realizada uma análise exploratória das porcentagens da cobertura da triagem auditiva neonatal nos quatro períodos. Nos mapas criados, a intensidade dos níveis de cinza está relacionada diretamente à magnitude das porcentagens, ou seja, quanto mais escuro, mais altos são os valores.

No quarto mapa foi realizada a análise de dependência espacial. Utilizou-se o índice de Moran Global que estima a autocorrelação espacial, podendo variar entre -1 e +1 , e fornece a significância estatística do mesmo (valor de p). Após a análise geral, foi avaliada a presença de clusters por meio do Moran local (Local Indicators of Spatial Association - LISA). Para tanto, foram construídos os BoxMap e MoranMap. O BoxMap apresenta os clusters, independente da significância estatística, enquanto o MoranMap apenas aqueles com valor de $\mathrm{p}<0,05$. Para a validação do índice de Moran Global foi utilizado o teste de permutação aleatória, com 99 permutações.

A medida de autocorrelação espacial sintetiza o relacionamento de um conjunto de unidades espaciais e valores de um atributo associados. Foram avaliados os padrões espaciais e classificados em agregados (clusters), dispersos ou aleatórios. Um valor de índice de Moran Global entre 0.1 e 1.0 indica um padrão de cluster não aleatório, entre -0.1 e 1.0 é dispersão e entre -0.1 e 0.1 é padrão aleatório.

Por fim, foi criada uma tabela com as modalidades de serviço de saúde auditiva do Brasil por Regiões (Norte, Nordeste, Sul, Sudeste e Centro-Oeste) para correlacionar com cobertura da triagem auditiva do último mapa. 


\section{Resultados}

A partir dos dados iniciais, observamos que a população de nascidos vivos ao longo do período estudado manteve-se praticamente constante e que a população beneficiária de plano de saúde vem aumentando discretamente, já a quantidade de triados teve um grande aumento no segundo e terceiro períodos. Observamos que a cobertura da triagem auditiva neonatal apresentou uma evolução de 9,3 para 37,2 \% (Tabela 1).
A análise espacial exploratória da porcentagem da cobertura da triagem está representada para as 161 Regiões Intermediárias de Articulação Urbana nos quatro mapas abaixo (Figura 1).

Em relação ao primeiro mapa da Figura 1 (jan/08 a dez/09), observa-se que a porcentagem da cobertura variou de 0,00 a $79,92 \%$, porém a maioria das regiões obteve cobertura entre $0,0 \mathrm{e}$ 19,99\%, 75 regiões tinham cobertura de $0,0 \%$ e as quatro regiões que apresentaram os melhores índices foram: Campina Grande (79,91\%), Ma-

Tabela 1. Distribuição dos nascidos vivos, beneficiários de plano de saúde, triados e cobertura no Brasil nos períodos estudados.

\begin{tabular}{lcccc}
\hline & jan/08-dez/09 & jan/10-dez/11 & jan/12-dez/13 & jan/14-jun/15 \\
\hline Nascidos vivos & 5.816 .409 & 5.775 .028 & 5.809 .816 & 4.356 .040 \\
Plano de saúde & 1.217 .003 & 1.287 .071 & 1.329 .346 & 1.422 .739 \\
Triados & 425.886 & 872.598 & 1.129 .871 & 1.090 .257 \\
Cobertura (\%) & 9,3 & 19,4 & 25,3 & 37,2 \\
\hline
\end{tabular}

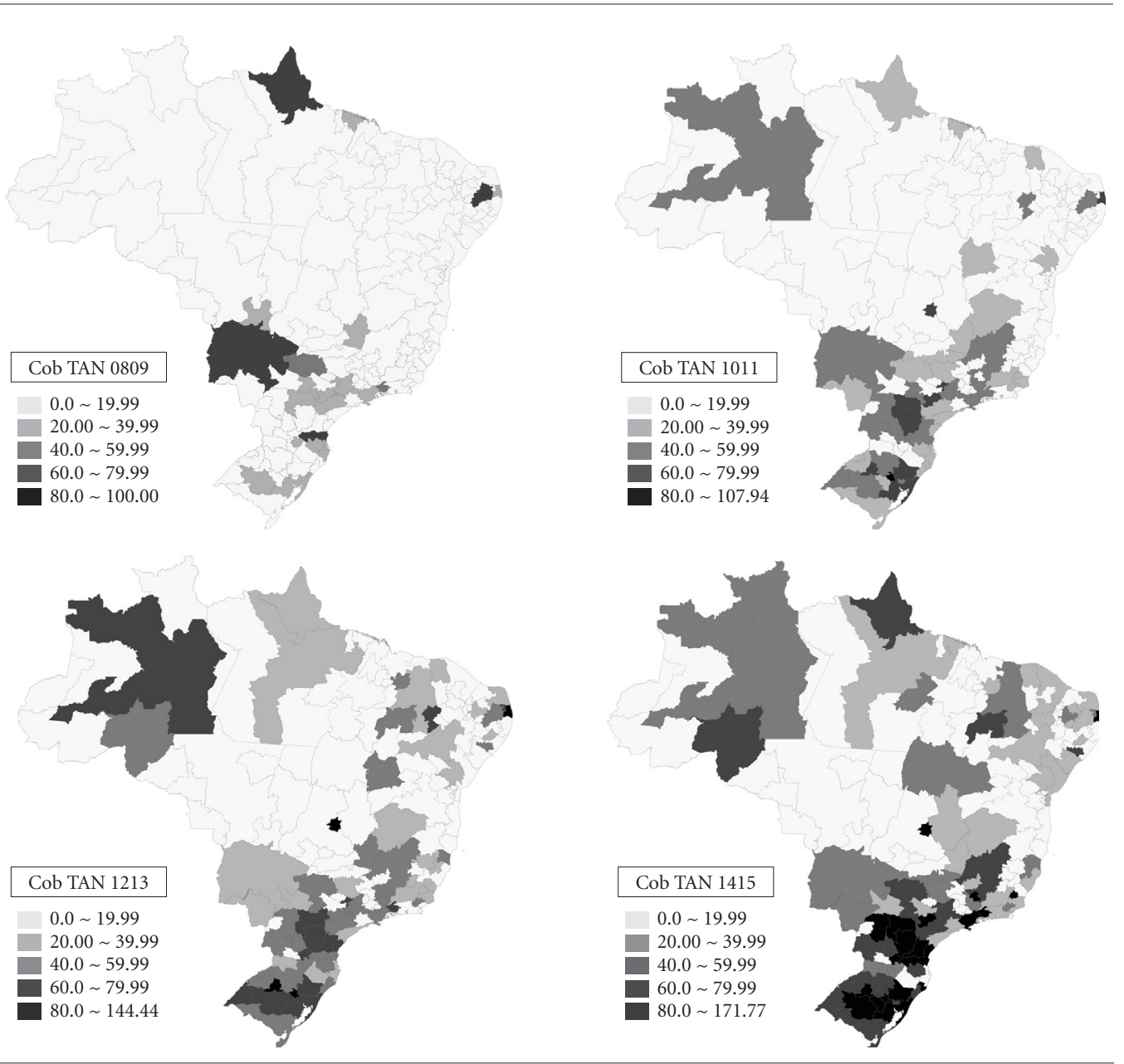

Figura 1. Distribuição espacial da porcentagem da cobertura da triagem auditiva neonatal nos períodos de 2008 a 2015 pelas Regiões Intermediárias de Articulação Urbana, Brasil. 
capá $(72,10 \%)$, Campo Grande (72,06\%) e Joinville $(60,15 \%)$.

Quanto ao segundo mapa da Figura 1 (jan/10 a dez/11), a cobertura variou de 0,0 a $107,94 \%$, com apenas uma região, Lajeado, obtendo cobertura maior que $95 \%$ e 48 regiões com cobertura de $0,0 \%$. Apareceram novas regiões, mas com predomínio nas regiões Sul e Sudeste.

No terceiro mapa da Figura 1 (jan/12 a dez/13), a cobertura variou de 0,0 a $144,44 \%$, demonstrando uma maior concentração quanti e qualitativa na região Sul e Sudeste. As quatro regiões que apresentam as melhores taxas foram: João Pessoa (89,74\%), Cruz Alta (84,92\%), Lajeado $(144,43 \%)$ e Anápolis $(81,53 \%)$. Também observa-se o surgimento de regiões e o aumento da porcentagem das que já apresentavam cobertura e a redução naquelas com cobertura de 0,0\%, de 48 para 34 .

Por fim, no último mapa da Figura 1 (jan/14 a jun/15) a cobertura variou entre 0,0 e 171,77\%, observa-se a manutenção das regiões, porém com um visível aumento da porcentagem da cobertura, principalmente na Região Sul e 28 regiões com cobertura de $0,0 \%$. Doze regiões apresentam cobertura maior que 95 \%, são elas: Lavras (97,7\%), Volta Redonda (110,78\%), Botucatu (140,39\%), Curitiba (119,02\%), Paranaguá (103,17\%), Joinville $(95,85 \%)$, Caxias do Sul $(131,02 \%)$, Cruz Alta (104,49\%), Lajeado (171,76\%), Porto Alegre (96,84\%), Santa Cruz do Sul (116,06\%) e Anápolis $(120,40 \%)$. Porém 27 regiões apresentam cobertura igual a zero, sendo estas concentradas nas Regiões Norte e Nordeste.

Podemos visualizar a evolução quantitativa da cobertura da triagem nas regiões na Tabela 2. Observamos a redução pela metade do número de regiões com cobertura entre 0,0 e 19,99\% do primeiro para o último período, porém ainda é a categoria predominante e que com o passar dos anos a cobertura da triagem foi redistribuída para outras, com maiores porcentagens.

Tabela 2. Distribuição das categorias de cobertura da TAN no Brasil nos períodos estudados.

\begin{tabular}{lcccc}
\hline $\begin{array}{c}\text { Cobertura } \\
\text { \% }\end{array}$ & jan/08-dez/09 & jan/10-dez/11 & jan/12-dez/13 & jan/14-jun/15 \\
\hline $0,0-19,99$ & $\mathbf{n}$ & $\mathbf{n}$ & $\mathbf{n}$ & $\mathbf{n}$ \\
$20,0-39,99$ & 138 & 105 & 85 & 70 \\
$40,0-59,99$ & 17 & 26 & 25 & 29 \\
$60,0-79,99$ & 2 & 20 & 31 & 21 \\
$>80,00$ & 4 & 9 & 16 & 21 \\
\hline
\end{tabular}

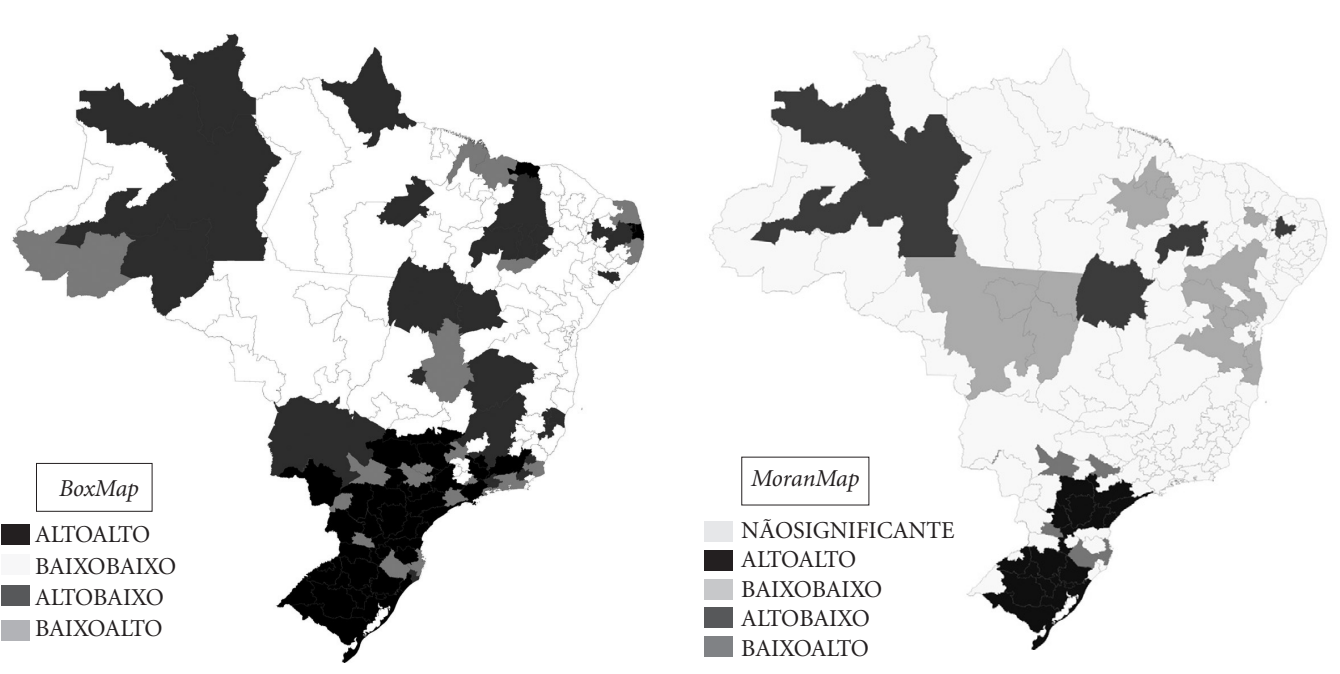

Figura 2. Distribuição espacial dos clusters das porcentagens da cobertura da triagem auditiva neonatal BoxMap e com LISA estatisticamente significativo MoranMap. 
Iniciando as análises estatísticas, foi calculado o Índice de Moran Global para a porcentagem da cobertura da triagem auditiva neonatal no período de janeiro de 2014 a junho de 2015 com valor de $0,397(\mathrm{p}=0,01)$, caracterizando um padrão de cluster disperso. Em seguida, foi realizada a análise local, a partir do Índice de Moran Local (LISA) e em seguida construídos os BoxMap e MoranMap (Figura 2).

$\mathrm{Na}$ análise de clusters, através do BoxMap, verifica-se a presença de um aglomerado Alto-Alto na Região Sul, nos três estados, e em algumas regiões dos estados de São Paulo e Minas Gerais, totalizando 43 Regiões Intermediárias de Articulação Urbana. Porém, na análise estatística, através do Moran Map, observamos que a maioria das regiões são não significantes. No estado do Rio Grande do Sul predominou o cluster Alto -Alto e em algumas regiões dos estados do Paraná e São Paulo este também foi significante.

Por fim, a Tabela 3 mostra a quantidade e a porcentagem de serviços de saúde auditiva, Centros de Implante Coclear, Centros de Média Complexidade, Centros de Alta Complexidade e Centros de Reabilitação de Modalidade Auditiva, a partir da Divisão Regional do Brasil.

Os Centros de Implante Coclear concentram-se na Região Sudeste, quase 50\% do total, enquanto a Região Norte apresenta apenas um serviço. Os serviços de Média e Alta Complexidade estão concentrados na Região Sudeste e escassos nas Regiões Norte e Centro-Oeste, porém a Região Sul apresenta uma distribuição próxima a do Sudeste em relação aos Centros de Média Complexidade. Quanto aos Centros de Reabilitação de Modalidade Auditiva observamos uma distribuição diferente dos demais serviços, pois a Região Sul apresenta a menor porcentagem, ficando a Região Sudeste com 47,1\%, seguida da Nordeste com 22,8\%.

\section{Discussão}

Os resultados do presente estudo demostram o crescimento significativo das taxas de cobertura da triagem auditiva neonatal no Brasil nesses últimos oito anos. Embora esse crescimento tenha ocorrido em nível nacional, destaca-se uma desigualdade inter-regional, pois as Regiões Intermediárias de Articulação Urbana das Regiões Sul e Sudeste concentram as melhores taxas. A taxa de cobertura nacional ainda é baixa $(37,2 \%)$ e muito inferior à recomendada pela literatura.

$\mathrm{O}$ aumento da cobertura da triagem no primeiro intervalo foi de $10,1 \%$, seguida de 5,9 e $11,9 \%$ no último estudado. Esses achados podem ser justificados pela Lei Federal 12.303, de 2 de agosto de 2010, que tornou obrigatória a realização gratuita do exame EOAE em todos os hospitais e maternidades, nas crianças nascidas em suas dependências ${ }^{5}$, pelo Decreto Federal 7.612 de 2011, que estabeleceu o Plano Nacional dos Direitos a Pessoa com Deficiência-Viver sem Limite, o qual criou a Rede de Cuidados à Pessoa com Deficiência e qualificou serviços de saúde auditiva e pela Portaria no 1.459 , de 24 de junho de 2011, que instituiu a Rede Cegonha e forneceu a recursos financeiros para compra de equipamento de triagem auditiva ${ }^{10}$. As porcentagens de cobertura dos dois primeiros períodos são bem próximas às encontradas na literatura ${ }^{8}$. Antes da lei federal, a maioria das regiões obteve uma baixa cobertura, mas com o decorrer dos anos essa cobertura foi aumentando, demonstrando o impacto positivo da lei. As regiões Campina Grande, Macapá, Campo Grande e Joinville que se destacaram com boas taxas no primeiro mapa, no segundo apresentam uma redução, provavelmente os serviços dessas regióes não estavam apoiados em legislações específicas para a triagem, tendo sido possíveis iniciativas de gestão local.

Tabela 3. Serviços de saúde auditiva segundo a Divisão Regional do Brasil.

\begin{tabular}{lcccccccc}
\hline \multirow{2}{*}{ Regiões } & \multicolumn{2}{c}{ Centros de IC } & \multicolumn{2}{c}{ Centros de MC } & \multicolumn{2}{c}{ Centros de AC } & \multicolumn{2}{c}{ CER MOD AUD } \\
\cline { 2 - 9 } & $\mathbf{n}$ & \multicolumn{1}{c}{$\boldsymbol{\%}$} & $\mathbf{n}$ & $\boldsymbol{\%}$ & $\mathbf{n}$ & \multicolumn{1}{c}{$\%$} & $\mathbf{n}$ & $\%$ \\
\hline Norte & 01 & 3,7 & 02 & 3,3 & 04 & 5,2 & 10 & 14,2 \\
Nordeste & 07 & 26,0 & 13 & 21,6 & 20 & 26,3 & 16 & 22,8 \\
Sudeste & 12 & 44,4 & 23 & 38,3 & 31 & 40,7 & 33 & 47,1 \\
Sul & 05 & 18,5 & 20 & 33,3 & 19 & 25 & 03 & 4,2 \\
Centro-Oeste & 02 & 7,4 & 02 & 3,3 & 02 & 2,6 & 08 & 11,4 \\
Total & 27 & 100 & 60 & 100 & 76 & 100 & 70 & 100 \\
\hline
\end{tabular}

NOTA: IC = Implante Coclear; MC = Média Complexidade; AC = Alta Complexidade; CER = Centros de Reabilitação; MOD = Modalidade; AUD = Auditiva. 
O surgimento de regiões e o aumento da porcentagem naquelas que já apresentavam cobertura entre os anos de 2012 e 2013 reforçam a ideia de que a implantação do programa de TANU pode ter ocorrido de forma gradativa, para melhor organização das ações necessárias. Entretanto, deve-se ter como meta a implementação da universalidade dessa triagem em um período de três anos ${ }^{6}$. Em contrapartida, ainda existem regiões com baixa cobertura. O nível de organização dos serviços de saúde pode influenciar positiva ou negativamente no registro do procedimento de triagem no SIA-SUS, e a ausência de dados, como observado em algumas regiões, não significa que necessariamente nenhum exame foi realizado.

Essa evolução da cobertura está de acordo com a bibliografia, que relata, entre 2004 e 2011, um aumento expressivo dos exames de emissões otoacústicas (EOA) para triagem auditiva ${ }^{11}$. Quanto ao número de regiões com cobertura maior que 95\%, antes da Lei Federal 12.303, de 2 de agosto de 2010, não existia região com essa taxa e nos dados mais recentes passou a ter doze regiões. Esses dados reforçam a importância das ações públicas sobre a triagem auditiva neonatal e o aumento da cobertura da triagem a nível nacional ${ }^{8}$. Tais achados corroboram com a literatura, segundo a qual menos do que a metade dos programas de triagem do Brasil conseguiu atingir o índice de $95 \%$ de recém-nascidos triados ${ }^{12}$.

As boas taxas de cobertura da triagem auditiva neonatal concentrada na Região Sudeste coincidem com a centralização de todas as diferentes modalidades de serviço de saúde auditiva e isso é resultado do pioneirismo na implantação da Política Nacional de Atenção à Saúde Auditiva ${ }^{13}$. A oferta profissional também pode contribuir, pois essa Região apresenta o maior número de fonoaudiólogos no país ${ }^{14}$.

$\mathrm{O}$ fato de nove regiões terem valores maiores que $100 \%$ entre os anos de 2014 e 2015 pode ser justificado pelo fato dessas regiões possuírem serviços de porte maior e triarem recém-nascidos de regiões vizinhas. Além disso, com a ausência do código para reteste no SIA-SUS o mesmo recém-nascido pode estar sendo contabilizado duas vezes ao ser submetido ao reteste, o que superestima o valor ${ }^{15}$ e/ou erro na alimentação do Sistema de Informações em Saúde ${ }^{16}$

A região Sul concentra oito regiões Intermediárias de Articulação Urbana com taxas maiores que $95 \%$. Esse dado corrobora a literatura, que relata que a região Sul apresenta maior uniformidade nas taxas, com seus três estados entre as sete maiores do país ${ }^{8}$. Outro fator que colabora é a alta concentração dos serviços de Média e Alta Complexidade, importantes porque podem realizar a triagem e dão prosseguimento as etapas seguintes dos programas? ${ }^{7}$.

No caso específico do estado do Rio Grande do Sul, duas cidades, Porto Alegre e Santa Maria, que estão entre as com melhores taxas, tiveram leis municipais aprovadas em 2004 que obrigavam a realização da triagem auditiva neonatal ${ }^{4}$. A legislação pioneira pode ter proporcionado uma melhor estruturação dos serviços resultando nas melhores taxas. No caso da região de Porto Alegre, esse achado coincide com a literatura ${ }^{17}$. As políticas locais do estado também corroboram com esses dados, pois no ano de 2006 foi criado o Programa Primeira Infância Melhor (PIM) que está presente em 267 municípios. Esse programa possui ações socioeducativas voltadas às famílias com crianças entre zero e seis anos e gestantes, que se encontram em situações de vulnerabilidade social. Esse Programa impactou positivamente na triagem auditiva do Rio Grande do Sul ${ }^{14}$.

O Paraná foi o primeiro estado brasileiro a legislar especificamente sobre a TAN, em 20014, e hoje apresenta duas regiões, Curitiba e Paranaguá, com as melhores taxas do Brasil. Em contrapartida, os estados de São Paulo e Pernambuco também foram pioneiros na legislação, porém no último período estudado não obtiveram regiões com as melhores coberturas. Percebe-se que somente a existência da lei não é suficiente para que seu cumprimento se efetive. É necessário que sejam garantidos os instrumentos e os recursos necessários para viabilizar a real implantação de um Programa de Triagem Auditiva Neonatal.

As piores taxas estão centradas nas Regiões do Norte e Nordeste, as quais não aparecem como pioneiras na legislação da triagem ${ }^{4}$ e fortalecem os achados da literatura, segundo a qual quase metades dos hospitais que não realizam a TAN se concentram nas regiões Centro-Oeste, Nordeste e Norte $^{18}$. Também são regiões mais extensas territorialmente e consequentemente podem possuir um acesso ao serviço difícil. A própria unidade de análise é baseada no fluxo de bens e serviços ${ }^{19}$.

Os poucos serviços de Média e Alta Complexidade nas Regiões Norte e Centro-Oeste corroboram com esses achados, pois comprometem o prosseguimento dos programas de TAN. Chama a atenção as Regiões Norte e Centro-Oeste quanto a uma melhor oferta dos Centros de Reabilitação de Modalidade Auditiva, provavelmente a política dessas Regiões deve estar voltada para 
crianças com comprometimentos associados (auditivo, físico, mental e motora).

Fora do eixo Sul e Sudeste, duas regiões obtiverem boas taxas de cobertura, João Pessoa $(94,50 \%)$ e Anápolis (120,40\%). No caso de João Pessoa, o dado corrobora com o achado do estado da Paraíba, que está entre os melhores índices do Brasil ${ }^{8}$. Esses dados reforçam a importância da unidade de análise estudada, visto que permitiu uma investigação mais minuciosa e fidedigna da cobertura da triagem no país.

Essa desigualdade inter-regional na cobertura da TAN no país foi verificada na análise estatística espacial, em que foi encontrado um padrão de cluster disperso com um aglomerado Alto-Alto na Região Sul e Sudeste e apenas o estado do Rio Grande do Sul e uma região entre os estados do Paraná e São Paulo permaneceram significativos estatisticamente, com o mesmo. Essa análise aponta uma diferença entre as regiões mais ricas e as mais pobres do país e fortalece os achados da literatura de que apesar do crescimento significativo da assistência fonoaudiológica no SUS, há uma má distribuição no território brasileiro ${ }^{8,12,20}$.

Através dessa análise constatamos que o Brasil não está cumprindo o indicador de universalidade do programa de TAN, pois a cobertura de janeiro de 2014 a junho de 2015 foi de 37,2\%, muito abaixo do recomendado pelo COMUSA $(2010)^{6}$. Apesar da publicação da Lei Federal sobre a obrigatoriedade da realização das EOE em maternidades e das recomendações do Ministério da Saúde para a sua realização. Esse dado não corrobora com os estudos internacionais, pois os serviços obtêm taxas entre 94,8 e 99,2\% $\%^{21,22}$.

O uso dos Sistemas de Informação em Saúde permitiu realizar a análise da evolução da cobertura da triagem auditiva neonatal no Brasil, apesar de existir muitos estudos sobre esses programas algumas não trazem esse dado. $\mathrm{O}$ uso dos sistemas permitiu analisar uma ampla cobertura populacional, teve baixo custo para a coleta das informações e a facilidade para o seguimento longitudinal, porém, como já foi dito, a falta da padronização na coleta dos dados pode levar ao o sub-registro ou a valores superestimados ${ }^{16}$.

Sabemos que a implantação de um programa de TAN, requer um investimento inicial e de manutenção, com o equipamento, o profissional, a ambiência e a necessidade de uma rede para encaminhamentos. Alguns dos motivos para hospitais não realizarem a TAN foram falta de local para encaminhamento no caso de falha, falta de conhecimento no assunto, baixo índice de nascimentos $^{18}$. O uso das Regiões Intermediárias de
Articulação Urbana permitiu uma análise mais fidedigna, pois podem existir municípios que não justifiquem a implantação de um programa, bem como ao contrário, regiões que deveriam ofertar uma maior quantidade de serviços.

Não há estudo epidemiológico sobre a perda auditiva neonatal no Brasil, por isso, atenção e esforços devem ser voltados para o desenvolvimento de um banco de dados nacional que contemple todos os aspectos do tratamento da criança de risco para perda auditiva, incluindo a triagem, o diagnóstico e a intervenção. Pois a importação de modelos e dados de outros países não atende as particularidades da nossa população e do nosso sistema de saúde.

A triagem auditiva neonatal é a precursora para alcançar o diagnóstico e a intervenção precoce da perda auditiva, e proporcionar qualidade de vida à criança ${ }^{1}$. Através de um banco de dados será possível realizar um levantamento da prevalência e da incidência da perda auditiva infantil no país, para consequentemente promover a vigilância epidemiológica e melhoria na qualidade da rede de atenção à saúde.

\section{Conclusão}

O Brasil apresenta uma evolução positiva na cobertura da triagem auditiva neonatal no período de janeiro de 2008 a junho de 2015, tendo como cobertura final $31,8 \%$. Observamos uma distribuição espacial desigual nas Regiões Intermediárias de Articulação Urbana, com as melhores coberturas concentradas nas Regiões Sul e Sudeste e consequentemente identificamos as regiões que precisam de mais esforços públicos para a implantação dos programas de TAN, que são as Regiões Norte e Nordeste.

A distribuição desigual da cobertura pode ser explicada pelas leis e políticas locais e pela disposição das diferentes modalidades de serviço de saúde auditiva, como é o caso das Regiões Intermediárias de Articulação Urbana da Região Sul, que conseguiram atingir a universalidade através de legislações e de uma rede de assistência à saúde bem articulada.

Por fim, sugere-se a criação do código do reteste da TAN pelo Ministério da Saúde no SIA-SUS, a fim de que as análises sejam mais fidedignas. As limitações do uso dos sistemas não inviabilizaram o estudo nem reduziram sua importância, pois foram consideradas na compreensão dos resultados. 


\section{Colaboradores}

MR Paschoal trabalhou na pesquisa e metodologia. HG Cavalcanti e MAF Ferreira contribuíram na redação final.

\section{Referências}

1. Lewis DR. Evidências para a Realização da Triagem Auditiva Neonatal Universal. In: Bevilacqua MC, Martinez MAN, Balen AS, Pupo AC, Reis ACM, Frota S, organizadores. Tratado de Audiologia. São Paulo: Ed. Santos; 2011. p. 495-513.

2. Yoshinaga-itano C, Sedey AL, Coulter DK, Mehl AL. Language of early-and later-identified children with hearing loss. American Academy of Pediatrics 1998; 102(5):1161-1171.

3. American Academy of Pediatrics. Task force on newborn and infant hearing. Newborn and Infant Hearing Loss: Detection and Intervention Pediatrics 1999; 103(2):527-530.

4. Tochetto T, Vieira EP. Legislação brasileira sobre triagem auditiva neonatal. Carapicuíba: Pró Fono; 2006.

5. Brasil. Lei no 12.303 , de 2 de agosto de 2010. I Dispõe sobre a obrigatoriedade de realização do exame denominado Emissões Otoacústicas Evocadas. Diário Oficial da União 2010; 03 out.

6. Lewis DR, Marone SAM, Mendes BCA, Cruz OLM, Nóbrega, M. Comitê Multiprofissional em Saúde Auditiva - COMUSA. Braz J Otorhinolaryngol 2010; 76(1):121-128.

7. Brasil. Ministério da Saúde (MS). Diretrizes de Atençãa da Triagem Auditiva Neonatal. Brasília: MS; 2012.

8. Cruz LRL, Ferrite, S. Cobertura estimada da triagem auditiva neonatal para usuários do Sistema Único de Saúde, Brasil, 2008-2011. Rev. Bras. Saúde Mater. Infant. 2014; 14(4):401-411.

9. Instituto Brasileiro de Geografia e Estatística (IBGE). Divisão Urbano Regional. Diretoria de Geociências Coordenação de Geografia. Rio de Janeiro: IBGE; 2013.

10. Brasil. Decreto no 7.612, de 17 de novembro de 2011 Instituiu o Plano Nacional dos Direitos da Pessoa com Deficiência - Plano Viver sem Limite. Diário Oficial da União 2011; 18 nov.

11. Silva LSG, Gonçalves CGO, Soares VMN. Política Nacional de Atenção à Saúde Auditiva: um estudo avaliativo a partir da cobertura de serviços e procedimentos diagnósticos. CoDAS [online] [periódico na Internet]. 2014 Jun. [acessado 2017 Maio 14]; 26(3): [cerca de 5 p.]. Disponível em: http://www. scielo.br/scielo.php?script=sci_isoref\&pid=S2317=17822014000300241-\&lng=en\&tlng$\mathrm{pt}$

12. Cavalcanti HG, Melo LPF, Buarque LFSFP, Guerra, RO. Overview of newborn hearing screening programs in Brazilian maternity hospitals. Braz J Otorhinolaryngol 2014; 80(4):346-353.

13. Manzoni CRCT, Almeida, SMVT. Organização da Rede de Atenção a Saúde Auditiva. In: Bevilacqua MC, Martinez MAN, Balen AS, Pupo AC, Reis ACM, Frota S, organizadores. Saúde auditiva no Brasil: políticas, serviços e sistemas. São José dos Campos: Pulso Editorial; 2010. p. 97-117.

14. Conselho Federal de Fonoaudiologia. Número de fonoaudiólogos no Brasil por Conselho Regional. [acessado 2015 Dez 10]. Disponível em: http://www.fonoaudiologia.org.br/cffa/index.php/numero-por-regiao/.

15. Fabrício MF. Avaliação da triagem auditiva neonatal no SUS: estudo da etapa do reteste em um grupo hospitalar de porto alegre e possíveis implicações para a gestão do sistema de saúde [dissertação]. Porto Alegre: Universidade Federal do Rio Grande do Sul; 2014. 
16. Coeli CM. Sistemas de Informação em Saúde e uso de dados secundários na pesquisa e avaliação em saúde. Cad Saúde Coletiva 2010; 18(3):335-336.

17. Canabarro MSM, Neli F, Vera WKM, Mitre EI. Programa de triagem auditiva neonatal: resultados de um Hospital Universitário de Porto Alegre. Revista HCPA 2012; 32(1):30-34.

18. Nunes CC. Caracterização dos Programas de Triagem Auditiva Neonatal no Brasil [dissertação]. São Paulo: Pontifícia Universidade Católica de São Paulo; 2013.

19. Instituto Brasileiro de Geografia e Estatística (IBGE). Diretoria de Geociências Coordenação de Geografia 2007. Regiões de influência das cidades. Rio de Janeiro: IBGE; 2008 .

20. Miranda GMD, Mendes ACG, Silva ALA, Rodrigues, M. Assistência fonoaudiológica no SUS: a ampliação do acesso e o desafio de superação das desigualdades. Rev. CEFAC 2015; 17(1):71-79.

21. Gilbey P, Kraus C, Ghanayim R, Sharabi-nov A, Bretler S. Universal newborn hearing screening in Zefat, Israel: The first two years. International Journal of Pediatric Otorhinolaryngology 2013; (77):97-100.

22. O'Connor A, O'Sullivan PG, Behan L, Norman G, Murphy B. Initial results from the newborn hearing screening programme in Ireland. Ir J Med Sci 2013; 182(4):551-555.

Artigo apresentado em 14/05/2016

Aprovado em 18/08/2016

Versão final apresentada em 20/08/2016 